\title{
BAM8 -22 Peptide Produces Itch and Nociceptive Sensations in Humans Independent of Histamine Release
}

\author{
Parul Sikand, ${ }^{1}$ Xinzhong Dong, ${ }^{2}$ and Robert H. LaMotte ${ }^{1}$ \\ ${ }^{1}$ Department of Anesthesiology, Yale University School of Medicine, New Haven, Connecticut 06520, and ${ }^{2}$ The Solomon S. Snyder Department of \\ Neuroscience, Center for Sensory Biology, School of Medicine, Johns Hopkins University, Baltimore, Maryland 21205
}

Chronic itch accompanying many dermatological, neurological, and systemic diseases is unresponsive to antihistamines. Our knowledge of endogenous chemicals that evoke histamine-independent itch and their molecular targets is very limited. Recently it was demonstrated in behavioral and cellular experiments that bovine adrenal medulla 8-22 peptide (BAM8 -22), a proteolytically cleaved product of proenkephalin $\mathrm{A}$, is a potent activator of Mas-related G-protein-coupled receptors (Mrgprs), MrgprC11 and hMrgprX1, and induces scratching in mice in an Mrgpr-dependent manner. To study the sensory qualities that BAM8-22 evokes in humans, we tested the volar forearm of 15 healthy volunteers with heat-inactivated cowhage spicules previously soaked in the peptide. BAM8-22 produced itch in each subject, usually accompanied by sensations of pricking/stinging and burning. The sensations were occasionally accompanied by one or more mechanically evoked dysesthesias, namely alloknesis, hyperknesis, and/or hyperalgesia, but no wheal or neurogenic flare in the skin surrounding the application site. The inactive truncated peptide BAM8-18 produced weak or no sensations. Pretreatment of the tested skin with an antihistamine cream (doxepin) inhibited histamine-induced sensations, dysesthesias, and skin reactions but not the sensations and dysesthesias evoked by BAM8-22. We show that BAM8-22 produces itch and nociceptive sensations in humans in a histamine-independent manner. Thus, BAM8-22 may be an endogenous itch mediator that activates, in humans, MrgprX1, a novel target for potential anti-itch treatments.

\section{Introduction}

The most well studied itch mediator, histamine, is released from mast cells to evoke itch and nociceptive sensations. Histamineinduced itch is accompanied by the presence of a wheal reaction, caused by the enhanced permeability of blood vessels, and a reddening of the skin (termed a "neurogenic flare") that is produced by an axonal reflex reaction. Though antihistamines block histamine-induced itch, they are typically ineffective in treating the itch accompanying many dermatological and systemic disorders such as atopic dermatitis and diseases of the liver and kidney.

The spicules of the tropical plant Mucuna pruriens, commonly known as cowhage, evoke itch and lesser nociceptive sensations (LaMotte et al., 2009) in a histamine-independent manner (Johanek et al., 2007) by releasing a cysteine protease capable of activating protease-activating receptors 2 and 4 (Reddy et al., 2008). Similar itch and nociceptive sensations are evoked by spicules previously soaked in capsaicin after first inactivating the pro-

Received March 8, 2011; revised April 4, 2011; accepted April 11, 2011.

Author contributions: P.S., X.D., and R.H.L. designed research; P.S. performed research; P.S. analyzed data; P.S. and R.H.L. wrote the paper.

This study was supported by the NINDS Grants P01 NS 047399 (to R.H.L.) and NS054791 (to X.D.). We thank Daniel Zelterman for his help with statistical analyses and Steven $G$. Shimada for valuable comments on the manuscript.

The authors declare no competing financial interests.

Correspondence should be addressed to either of the following: Dr. Robert H. LaMotte, Department of Anesthesiology, Yale University School of Medicine, 333 Cedar Street, New Haven, CT 06520, E-mail: robert.lamotte@yale.edu; or Dr. Xinzhong Dong, The Solomon S. Snyder Department of Neuroscience, Center for Sensory Biology, School of Medicine, Johns Hopkins University, Baltimore, MD 21205, E-mail: xdong2@jhmi.edu.

DOI:10.1523/JNEUROSCI.1192-11.2011

Copyright $\odot 2011$ the authors $\quad 0270-6474 / 11 / 317563-05 \$ 15.00 / 0$ tease by heat (Sikand et al., 2009). However, no efficient clinical drug is available to treat chronic itch because of our limited knowledge of the molecular/cellular targets involved in mediating histamine-independent itch.

The family of Mas-related G-protein-coupled receptors (Mrgprs) consists of $>50$ members of which the mouse MrgprA, MrgprB, MrgprC, and MrgprD subfamilies are exclusively expressed in the small diameter sensory neurons in the DRG and trigeminal ganglia (Dong et al., 2001; Zylka et al., 2003). In humans, MrgprX1 shares sequence homology with the mouse MrgprA and MrgprC subfamilies and is restricted to small diameter DRG neurons (Lembo et al., 2002). Using in vitro binding assays, Lembo et al. (2002) demonstrated that the proteolytically cleaved product of proenkephalin A, the bovine adrenal medulla peptide 8-22 (BAM8-22), potently activated the hMrgprX1 by an opioidindependent mechanism. In contrast, BAM8-18, an identical peptide but lacking the last four amino acids, had no biological effect on hMrgprX1.

BAM8-22 evoked a calcium influx in HEK cells stably transfected with hMrgprX1 or murine MrgprC11 (Liu et al., 2009). In addition, a small subset of DRG neurons was activated by BAM8 -22 in an Mrgpr-dependent manner (Liu et al., 2009). Injections of BAM8-22 in the nape of the neck of wildtype mice produced significantly stronger scratching responses than in Mrgpr-deficient mice, suggesting a possible role of this ligand and its receptor in mediating itch (Liu et al., 2009).

Here we show that BAM8-22 evokes itch, nociceptive sensations, and cutaneous dysesthesias in human subjects by a histamineindependent mechanism. 


\section{Materials and Methods}

A total of 15 healthy subjects, 13 females and two males, aged 19-62 years, participated in the experiments. Subjects presenting a history of dermatological, neurological, immunological, or cardiac disorders were excluded. Additionally, subjects were asked to refrain from taking antihistamines and analgesics at least $24 \mathrm{~h}$ before an experiment. The Yale University Human Investigation Committee approved all protocols.

Cowhage spicules rendered chemically inert by autoclaving were soaked in $0.004,0.04,0.4$, or $4 \mathrm{mg} / \mathrm{ml}$ BAM8-22 solution (Tocris Bioscience); $3 \mathrm{mg} / \mathrm{ml}$ of the putatively inactive peptide, BAM8 -18 (synthesized in the Keck Lab, Yale University, New Haven, CT); or $10 \mathrm{mg} / \mathrm{ml}$ histamine solution (Sigma), each prepared in distilled water. Using a stereomicroscope, the spicules were observed as they filled. The filled spicules were pulled out of the chemical solution and allowed to dry on a filter paper placed in a Petri dish. Based on calculations described by Sikand et al. (2009), the estimated maximal amount of BAM8-22 available to the skin in the inserted tip of a spicule was $0.12,1.2$, or $12 \mathrm{pg}$ for a single spicule soaked in solutions of $0.004,0.04$, or $0.4 \mathrm{mg} / \mathrm{ml}$, respectively; 120 $\mathrm{pg}$ for the solution of $4 \mathrm{mg} / \mathrm{ml}$; and $90 \mathrm{pg}$ for a spicule soaked in $3 \mathrm{mg} / \mathrm{ml}$ BAM8-18.

The subjects were trained to rate the perceived intensity of itch, pricking/stinging, and burning evoked by a given stimulus by using the generalized labeled magnitude scale (Green et al., 1996). Itch was defined as a sensation that elicits a desire to scratch. Pricking/stinging was described as a sharp, localized sensation similar to a prick from a needle (intermittent in duration) or sting from being bitten by an insect (continuous in duration). Burning was defined as a sensation that most often accompanies thermal burns and sunburns but may be induced by other stimuli such as chemical irritants, skin abrasions, or strong cold. The subjects were instructed that the sensations of pricking/stinging and burning may or may not be painful. The subjects were asked to disregard the initial prick accompanying the insertion of the spicules in the skin. They were also instructed to only rate the sensations they experienced and that some stimuli may produce no sensations. In rapid sequence (within 20-30 s), the tips of three spicules containing either BAM8-22 or BAM8-18 were inserted obliquely into the skin $\sim 0.2 \mathrm{~mm}$ by means of forceps, under a stereomicroscopic view (LaMotte et al., 2009; Sikand et al., 2009).

To test for the contribution of histamine to BAM8-22-induced sensations and to any evoked wheal or flare, a topical antihistamine cream (Prudoxin, containing 5\% doxepin hydrochloride; Healthpoint) or a placebo cream (Vanicream; Pharmaceutical Specialties) was applied to the skin for $1.5 \mathrm{~h}$ before the application of spicules containing BAM $8-22$ or histamine. The antihistamine and the placebo cream contained similar inactive ingredients. Two adhesive patches $4 \times 4 \mathrm{~cm}(1 \mathrm{~mm}$ thick, foam adhesive tape; $3 \mathrm{M}$ ), each with a $3.5 \times 3.5 \mathrm{~cm}$ center cut out (to deposit $1.2 \mathrm{~g}$ of either cream) were placed $3 \mathrm{~cm}$ distal and $3 \mathrm{~cm}$ proximal to the center of the volar aspect of each arm. Each patch was covered with Tegaderm I.V., an occlusive adhesive dressing (3M), for at least $1.5 \mathrm{~h}$. The site of application of the antihistamine and the placebo was randomly chosen with the restriction that the two distal sites and the two proximal sites always received the same treatment. At the end of the pretreatment period, the patches were removed and the skin was cleaned with alcohol before testing with spicules. Three spicules of either histamine or BAM8 -22 $(4 \mathrm{mg} / \mathrm{ml})$ were applied in a randomized order such that each stimulus had been tested once within a site pretreated with antihistamine or placebo cream.

The borders of any areas of hypersensitivity to mechanical stimuli (dysesthesia), consisting of alloknesis, hyperknesis, and/or hyperalgesia, were mapped and marked on the skin at the end of sensory ratings, as described previously (LaMotte et al., 2009; Sikand et al., 2009).

If present, the border of a wheal (raised edematous region) and/or a visible redness (neurogenic flare) was marked. Occasionally, a small area of redness of $\sim 1-2 \mathrm{~mm}$ accompanied the application of spicules. A flare reaction $>1 \mathrm{~cm}^{2}$ in area was included in the analyses. The areas of skin reaction and dysesthesias were marked and photographed using a Sony digital camera.

For each sensory quality, the following parameters were calculated from psychophysical functions relating the perceived intensity versus

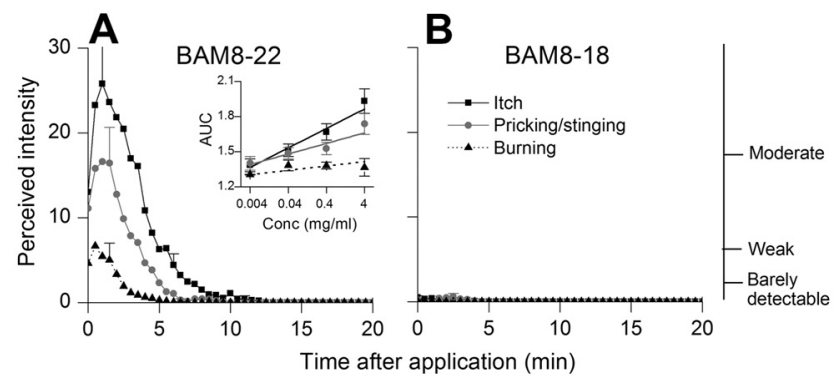

Figure 1. Time course of the mean perceived intensity of itch and nociceptive sensations evoked by spicules containing BAM8 -22 or the inactive peptide BAM8-18. $A, B$, The mean ratings of itch, pricking/stinging, and burning obtained from 15 subjects are plotted for successive intervals of 30 s following spicule application. Starting with the peak rating of each sensory quality, the SEM is plotted every $5 \mathrm{~min}$. The right vertical axis marks the position of three verbal descriptors shown in correspondence with the ratings of mean perceived intensity indicated on the left vertical axis. Inset, The linear regressions for concentration and the logarithmic values of the AUC for itch, pricking/stinging, and burning. Significant slopes were obtained for itch and pricking/stinging but not for burning. Conc, Concentration.

time from the onset of spicule insertion: the area under the curve (AUC), the peak magnitude of sensation, and the total duration of the sensation from the first nonzero value (rated greater than the category "barely detectable" on the scale) to the first of the three consecutive zeros or until $20 \mathrm{~min}$ had elapsed. Only sensory qualities rated greater than barely detectable were included in the analyses.

Linear regression lines were computed for different concentrations of BAM8-22 and the log value of each parameter. The parameter was determined to depend on the concentration if the slope of the line was significantly different from zero. Student's $t$ test was used to test the significance of differences in sensory rating measures, areas of dysesthesias, or areas of skin reactions between the two treatment groups. The significance of differences in the incidence of a given dysesthesia, wheal, or flare in skin treated with the placebo versus the antihistamine were determined using Fischer's exact test. The marked areas of dysesthesias and skin reaction in the digitized pictures were measured using Image software (NIH).

All statistical calculations were performed using the GraphPad Prism 4.03 software (GraphPad Software). The data are presented as means \pm SEMs and the probability ( $p$ ) value for statistical significance was $<0.05$.

\section{Results}

\section{Pruritic and nociceptive sensations induced by BAM8-22 and BAM8-18}

The BAM8-22 spicules elicited itch accompanied by pricking/ stinging and burning (Fig. $1 A$ ). The temporal profile of the mean perceived intensity was similar for each quality of sensation, peaking within the first minute after application and decreasing slowly over time. All subjects tested with BAM8-22 experienced itch. Fourteen also rated sensations of pricking/stinging and eight reported burning. Itch and pricking/stinging were the predominant sensations and were generally rated significantly higher than the sensation of burning.

BAM8-18 spicules produced a transient itch of a minute or less in only four subjects. In an additional subject, the sensation lasted for $4.5 \mathrm{~min}$. The peak magnitude of itch reported by these subjects was significantly lower than the peak itch they experienced with BAM8-22. Transient and weak sensations were reported as pricking/stinging in nine subjects and as burning in one. For subjects reporting sensations to either peptide, the mean value obtained for each sensory measure of itch was significantly greater for BAM8-22 than BAM8-18 (AUC: $102.4 \pm 22.2$ vs $3.5 \pm 2, p=0.02$; peak magnitude: $32.2 \pm 6.7$ vs $2.2 \pm 0.5, p=$ 0.02 ; total duration in minutes: $7.1 \pm 0.8$ vs $1.7 \pm 0.8, p=0.0012$; 


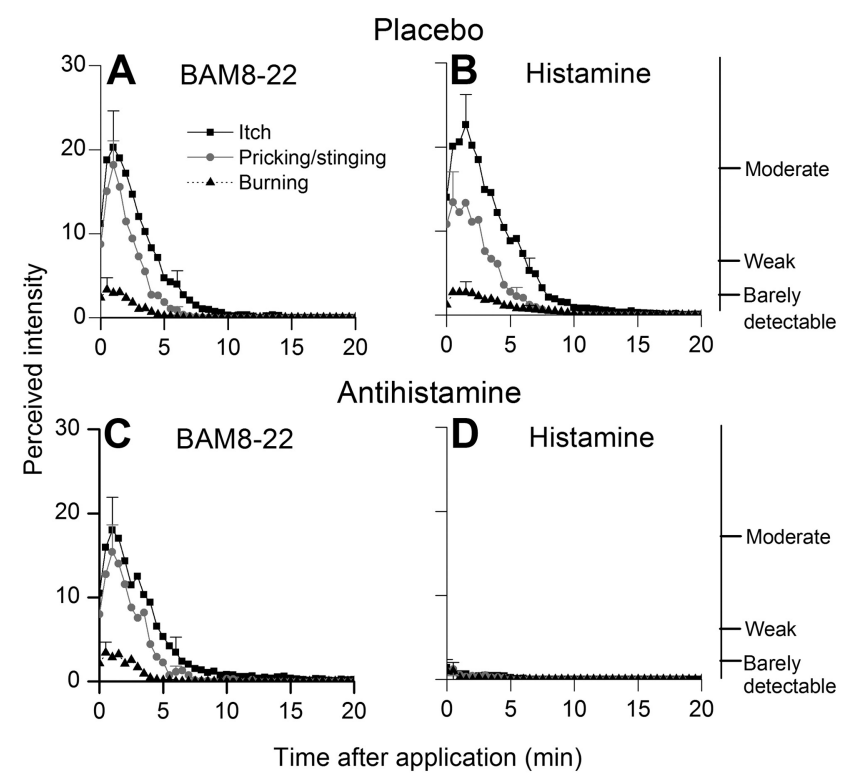

Figure 2. $\boldsymbol{A}-\boldsymbol{D}$, Time course of the perceived intensity of itch and nociceptive sensations evoked by spicules containing BAM8 $-22(\boldsymbol{A}, \boldsymbol{C})$ or histamine $(\boldsymbol{B}, \boldsymbol{D})$ applied to skin pretreated with an antihistamine $(\boldsymbol{C}, \boldsymbol{D})$ or a placebo $(\boldsymbol{A}, \boldsymbol{B})$ in a topical cream. The antihistamine inhibited the sensations evoked by histamine but not by BAM8 -22 .
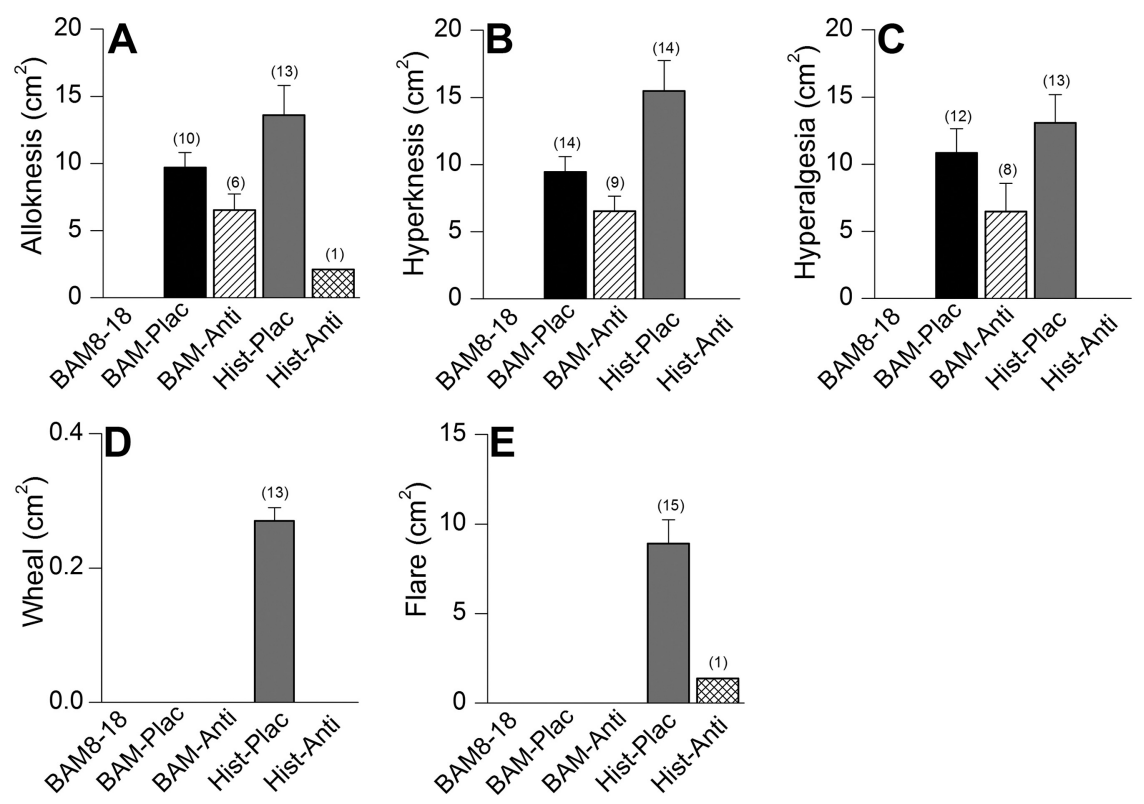

Figure 3. The effects of an antihistamine on the mean areas of dysesthesia, wheal, and flare evoked by spicules containing BAM8 -22 or histamine. $\boldsymbol{A}-\boldsymbol{E}$, The mean areas of alloknesis $(\boldsymbol{A})$, hyperknesis $(\boldsymbol{B})$, hyperalgesia $(\boldsymbol{C})$, wheal $(\boldsymbol{D})$, and flare $(\boldsymbol{E})$ evoked by BAM8-22 (BAM) or histamine (Hist) applied to skin pretreated with a topical cream containing placebo (Plac) or an antihistamine (Anti). The number of subjects reporting each effect is in parentheses. The antihistamine prevented or significantly attenuated only the sensory effects of histamine. BAM8 -22 produced no wheal or flare. BAM8-18 evoked no dysesthesias, wheal, or flare.

Student's $t$ test). Similarly, BAM8-22 elicited greater mean values for each measure of pricking/stinging (AUC: $53.6 \pm 11.9$ vs $2 \pm 0.6, p=0.002$; peak magnitude: $22.2 \pm 4.8$ vs $2.7 \pm 0.5, p=$ 0.004 ; total duration in minutes: $6.2 \pm 0.6$ vs $0.9 \pm 0.3, p<$ 0.0001 ). The incidence of burning with BAM8 -18 was too low to permit similar statistical comparisons.

Linear regression lines were used to study the relationship between the concentration of BAM8-22 and the mean logarith- mic value of the AUC, peak magnitude, and duration of sensations in 12 subjects. The slopes of the regression lines for AUC (Fig. 1, inset) and duration were significantly different from zero for itch $(p<0.0001$ and $p=0.02)$ and pricking/stinging ( $p=$ 0.0006 and $p=0.0048)$ but not for burning $(p=0.79$ and $p=$ 0.09 ). The peak magnitude of itch ( $p=0.001)$, pricking/stinging ( $p=0.0048$ ), and burning ( $p=0.02$ ) were also determined to be dependent on the concentration of BAM8-22. Thus, the magnitude and duration of itch and pricking/stinging increased with the concentration of BAM8-22.

\section{Effects of antihistamine on pruritic and nociceptive sensations}

Pretreatment with the antihistamine inhibited the itch and nociceptive sensations evoked by histamine but had no effect on the sensations evoked by BAM8-22 (Fig. 2). Comparable values of AUC, peak magnitude, total duration of itch, pricking/stinging, and burning evoked by BAM8 -22 were obtained in placebo and antihistamine-pretreated skin (Student's $t$ test, $p>0.05$ ). For example, the mean AUC of itch with BAM8-22 was $74.1 \pm 18.1$ in placebo-pretreated skin and $76 \pm 19$ after treatment with antihistamine.

In contrast, the antihistamine significantly attenuated the sensations elicited by histamine. The area under the curve, peak magnitude, total duration of itch, and pricking/stinging evoked by histamine were significantly decreased after antihistamine-pretreatment when compared with sensations evoked in placebopretreated skin (Student's $t$ test, $p<0.05$ ). For example, the mean AUC of itch with histamine in placebo-treated skin was $107 \pm 14.9$ and in antihistamine-treated skin was only $8.6 \pm 6$.

\section{Effects of antihistamine on dysesthesias and skin reactions}

No dysesthesias were reported in response to the inactive peptide, BAM8-18. In contrast, both BAM8-22 and histamine elicited one or more dysesthesias, namely alloknesis, hyperknesis, and/or hyperalgesia. The antihistamine had no significant effect on the areas (Student's $t$ test, $p>$ $0.05)$ and incidence of occurrence (Fischer's exact test) of alloknesis ( $p=0.27$ ), hyperknesis $(p=0.08)$, or hyperalgesia $(p=0.25)$ evoked by BAM8-22. It cannot be predicted whether these effects would reach significance if the power to detect significance was increased with a much larger sample size. In contrast, the effects of antihistamine were much greater and reached significance in decreasing the areas and occurrence of alloknesis $(p<$ $0.0001)$, hyperknesis $(p<0.0001)$, and hyperalgesia $(p<0.0001)$ elicited by histamine (Fig. $3 A-D$ ). For example, the areas and occurrence of alloknesis elicited by histamine in placebo-pretreated skin was $10.9 \pm 1.8 \mathrm{~cm}^{2}$ and $87 \%$, and decreased to $2.1 \mathrm{~cm}^{2}$ and $6.7 \%$, respectively, after pretreatment with antihistamine.

Neither BAM8-18 nor BAM8-22 evoked a wheal or neurogenic flare. For histamine, the antihistamine inhibited the formation of a wheal and significantly decreased the areas and 
occurrence of neurogenic flare $(p<0.0001)$ (Fig. $3 D, E)$. The incidence of occurrence and the mean area of the flare decreased respectively from $100 \%$ and $8.8 \pm 1.3 \mathrm{~cm}^{2}$, respectively, after the placebo treatment to $6.7 \%$ and $1.4 \mathrm{~cm}^{2}$ after treatment with the antihistamine.

\section{Discussion}

The central finding is that punctate superficial application of BAM8-22 evokes pruritic and nociceptive sensations that are not blocked by an antihistamine. The inactive, truncated peptide, BAM8-18, elicited either no sensations or sensations that were weak and transient. Thus, the sensory responses induced by BAM8-22 may be due to the activation of its specific receptor, hMrgprX1. This is consistent with hypothesis that there is a distinct class of sensory neurons mediating nonhistaminergic itch.

Recent studies have found the expression of a unique family of G-protein coupled receptors, the Mrgprs. A subset of these receptors, the murine MrgprAs, MrgprDs, MrgprC11, and the hMrgprX1, are exclusively expressed in a subset of small diameter cutaneous sensory neurons that are implicated in the transmission and modulation of itch and pain sensations (Dong et al., 2001; Lembo et al., 2002). MrgprA and MrgprC11 are coupled to $\mathrm{G} \alpha_{\mathrm{q} / 11}$ proteins (Han et al., 2002). The hMrgprX1-mediated calcium mobilization in transfected HEK cells was reported to be unaffected by pertussis toxin pretreatment, suggesting involvement of $\mathrm{G} \alpha$ protein (Lembo et al., 2002).

BAM8-22 is a proteolytically cleaved product of proenkephalin $\mathrm{A}$ and has been detected in various human tissues (Ferri et al., 1988). BAM8-22 lacks the opioid motif, YGGFM (metenkephalin), and hence, does not bind to the opioid receptors (Lembo et al., 2002). It is implicated in pain transmission as evidenced, for example, by its intrathecal administration that attenuated inflammatory hyperalgesia in mice by an Mrgprdependent mechanism (Guan et al., 2010). In cellular experiments, BAM8-22 has been reported to activate hMrgprX1 by an opioid-independent pathway (Lembo et al., 2002) and mouse MrgprC11 (Liu et al., 2009).

In cellular experiments, sensory neurons of mice expressing MrgprA3 responded to histamine and coexpressed MrgprC11 and the itch-mediating neuropeptide, gastrin-releasing peptide (Liu et al., 2009). MrgprC11 and MrgprA3 share a sequence homology that closely resembles hMrgprX1 (Dong et al., 2001). In vitro experiments established BAM8-22 as a potent agonist of MrgprC11. Additionally, administration of BAM8-22 in mice induced scratching behavior (Liu et al., 2009). Thus, murine MrgprA3 and MrgprC11 and hMrgprX1 may have a role in mediating itch.

One challenge in studies of behavioral responses elicited by potential pruritogens in animals only is that some chemicals such as serotonin that elicit scratching responses in mice may produce only weak itch when tested in humans (Schmelz et al., 2003). Thus, it is imperative that substances/chemicals that produce scratching in animals be tested in humans.

How does BAM8-22 evoke itch in humans? One possible explanation is that BAM8-22 causes a release of histamine from mast cells. The role of histamine in BAM8-22-induced scratching behavior in mice has not been studied. The antimalarial drug, chloroquine, produces itch in most black Africans as a side effect. Its intradermal administration in mice degranulates mast cells, partly contributing to the observed scratching behavior (Liu et al., 2009). In the present study, the application of antihistamine did not affect BAM8-22-induced itch but inhibited histamineevoked itch. The amount of antihistamine applied and the duration of application were sufficient to significantly inhibit the itch, flare, and dysesthesias induced by histamine. We conclude that BAM8-22 induces itch independent of a histaminergic mechanism.

Chemically soaked heat-inactivated cowhage spicules provide the most efficient and adequate means of intradermal application of different doses of chemicals to superficial pruriceptive nerve terminals (Sikand et al., 2009), including the delivery of substances such as cysteine proteases, which do not elicit a reliable itch when delivered by intradermal injection (Simone et al., 1987) but do when applied by spicule (Reddy et al., 2008, 2010). Since application of an algogen like capsaicin via spicules evokes itch and nociceptive sensations (Sikand et al., 2009) similar to those produced by cowhage, cathepsin S, and BAM8-22 spicules, further studies are needed to establish whether these chemicals are predominantly pruritic if administered by intradermal injections.

Cowhage spicules produce a histamine-independent itch in the absence of flare by activating mechanically sensitive afferents (MSAs) in humans and primates (Johanek et al., 2008; Namer et al., 2008). Histamine spicules or injections produce itch accompanied by flare by activating MSAs in primates (Ringkamp et al., 2010) and mechanically insensitive afferents (MIAs) in humans (Schmelz et al., 1997). MIA fibers have been previously reported to produce flare upon activation (Schmelz et al., 2000). Since a flare did not accompany pruritic and nociceptive sensations induced by BAM8-22, it can be hypothesized that, when applied via spicules, BAM8-22 activates mechanically sensitive nociceptive neurons.

Endogenous peptides like substance $\mathrm{P}$, vasoactive intestinal peptide, calcitonin gene-related peptide, serotonin, neuropeptide $\mathrm{Y}$, neurotrophins such as nerve growth factor, proteases, and interleukins have been implicated in itch and associated nociceptive sensations (Steinhoff et al., 2006). However, the underlying mechanisms by which these chemicals mediate itch are unclear. Substance $P$ has been reported to produce itch in humans mainly by degranulating mast cells to release histamine (Hägermark et al., 1978; Amatya et al., 2010). A wheal and flare accompanied the itch evoked by intradermal injections of substance P in these studies. Thus, our knowledge of endogenous itch mediators that may be implicated in producing most types of chronic, histamine-independent itch is very limited. A potential candidate is the endogenous cysteine protease Cathepsin S, recently reported to elicit predominant sensation of itch accompanied by lesser nociceptive sensations in the absence of wheal or flare in human subjects (Reddy et al., 2010). Another candidate could be the BAM8-22 peptide, which our results suggest can elicit itch in humans without releasing histamine. Therefore, BAM8-22 may be used as an important tool to selectively activate and study nerve fibers that mediate histamine-independent itch.

The amount of BAM8-22 in human skin has not been specifically determined. However, the expression of its precursor, proenkephalin A, along with Leu- and Met-enkephalin peptides, was observed in human skin with significant expression in the fibroblasts and keratinocytes (Slominski et al., 2011). Furthermore, the expression of these peptides was reported to be upregulated or altered in pathological conditions of the skin like psoriasis, squamous cell carcinoma, lichenoid, and seborrheic keratosis (Slominski et al., 2011). Thus, it is plausible that BAM8-22 is expressed in human skin and may activate pruriceptive nerve fibers via hMrgprXl, thereby contributing to the chronic itch accompanying many dermatological disorders. 


\section{References}

Amatya B, Nordlind K, Wahlgren CF (2010) Responses to intradermal injections of substance $\mathrm{P}$ in psoriasis patients with pruritus. Skin Pharmacol Physiol 23:133-138.

Dong X, Han S, Zylka MJ, Simon MI, Anderson DJ (2001) A diverse family of GPCRs expressed in specific subsets of nociceptive sensory neurons. Cell 106:619-632.

Ferri GL, Watkinson A, Dockray GJ (1988) Proenkephalin A-derived peptides in the human gut. Gastroenterology 95:1011-1017.

Green BG, Dalton P, Cowart B, Shaffer G, Rankin K, Higgins J (1996) Evaluating the 'Labeled Magnitude Scale' for measuring sensations of taste and smell. Chem Senses 21:323-334.

Guan Y, Liu Q, Tang Z, Raja SN, Anderson DJ, Dong X (2010) Mas-related G-protein-coupled receptors inhibit pathological pain in mice. Proc Natl Acad Sci U S A 107:15933-15938.

Hägermark O, Hökfelt T, Pernow B (1978) Flare and itch induced by substance P in human skin. J Invest Dermatol 71:233-235.

Han SK, Dong X, Hwang JI, Zylka MJ, Anderson DJ, Simon MI (2002) Orphan $\mathrm{G}$ protein-coupled receptors MrgA1 and $\mathrm{MrgC} 11$ are distinctively activated by RF-amide-related peptides through the Galpha q/11 pathway. Proc Natl Acad Sci U S A 99:14740-14745.

Johanek LM, Meyer RA, Hartke T, Hobelmann JG, Maine DN, LaMotte RH, Ringkamp M (2007) Psychophysical and physiological evidence for parallel afferent pathways mediating the sensation of itch. J Neurosci 27:7490-7497.

Johanek LM, Meyer RA, Friedman RM, Greenquist KW, Shim B, Borzan J, Hartke T, LaMotte RH, Ringkamp M (2008) A role for polymodal C-fiber afferents in nonhistaminergic itch. J Neurosci 28:7659-7669.

LaMotte RH, Shimada SG, Green BG, Zelterman D (2009) Pruritic and nociceptive sensations and dysesthesias from a spicule of cowhage. J Neurophysiol 101:1430-1443.

Lembo PM, Grazzini E, Groblewski T, O’Donnell D, Roy MO, Zhang J, Hoffert C, Cao J, Schmidt R, Pelletier M, Labarre M, Gosselin M, Fortin Y, Banville D, Shen SH, Ström P, Payza K, Dray A, Walker P, Ahmad S (2002) Proenkephalin A gene products activate a new family of sensory neuron-specific GPCRs. Nat Neurosci 5:201-209.

Liu Q, Tang Z, Surdenikova L, Kim S, Patel KN, Kim A, Ru F, Guan Y, Weng HJ, Geng Y, Undem BJ, Kollarik M, Chen ZF, Anderson DJ, Dong X
(2009) Sensory neuron-specific GPCR Mrgprs are itch receptors mediating chloroquine-induced pruritus. Cell 139:1353-1365.

Namer B, Carr R, Johanek LM, Schmelz M, Handwerker HO, Ringkamp M (2008) Separate peripheral pathways for pruritus in man. J Neurophysiol 100:2062-2069.

Reddy VB, Iuga AO, Shimada SG, LaMotte RH, Lerner EA (2008) Cowhageevoked itch is mediated by a novel cysteine protease: a ligand of proteaseactivated receptors. J Neurosci 28:4331-4335.

Reddy VB, Shimada SG, Sikand P, Lamotte RH, Lerner EA (2010) Cathep$\sin \mathrm{S}$ elicits itch and signals via protease-activated receptors. J Invest Dermatol 130:1468-1470.

Ringkamp M, Borzan J, Schaefer K, Hartke TV, Meyer RA (2010) Activation of polymodal nociceptors in monkey by punctate chemical stimulation with histamine and capsaicin. Soc Neurosci Abstr 36:584.6

Schmelz M, Schmidt R, Bickel A, Handwerker HO, Torebjörk HE (1997) Specific C-receptors for itch in human skin. J Neurosci 17:8003-8008.

Schmelz M, Michael K, Weidner C, Schmidt R, Torebjörk HE, Handwerker HO (2000) Which nerve fibers mediate the axon reflex flare in human skin? Neuroreport 11:645-648.

Schmelz M, Schmidt R, Weidner C, Hilliges M, Torebjork HE, Handwerker HO (2003) Chemical response pattern of different classes of C-nociceptors to pruritogens and algogens. J Neurophysiol 89:2441-2448.

Sikand P, Shimada SG, Green BG, LaMotte RH (2009) Similar itch and nociceptive sensations evoked by punctate cutaneous application of capsaicin, histamine and cowhage. Pain 144:66-75.

Simone DA, Ngeow JY, Whitehouse J, Becerra-Cabal L, Putterman GJ, LaMotte RH (1987) The magnitude and duration of itch produced by intracutaneous injections of histamine. Somatosens Res 5:81-92.

Slominski AT, Zmijewski MA, Zbytek B, Brozyna AA, Granese J, Pisarchik A, Szczesniewski A, Tobin DJ (2011) Regulated proenkephalin expression in human skin and cultured skin cells. J Invest Dermatol 131:613-622.

Steinhoff M, Bienenstock J, Schmelz M, Maurer M, Wei E, Biró T (2006) Neurophysiological, neuroimmunological, and neuroendocrine basis of pruritus. J Invest Dermatol 126:1705-1718.

Zylka MJ, Dong X, Southwell AL, Anderson DJ (2003) Atypical expansion in mice of the sensory neuron-specific Mrg G-protein-coupled receptor family. Proc Natl Acad Sci U S A 100:10043-10048. 